\title{
छூ \\ Back-bombardment compensation in microwave thermionic electron guns
}

\author{
Jeremy M. D. Kowalczyk ${ }^{*}$ and John M. J. Madey \\ Department of Physics and Astronomy, University of Hawaii Mānoa, Honolulu, Hawaii 96822, USA
}

(Received 23 July 2014; published 8 December 2014)

\begin{abstract}
The development of capable, reliable, and cost-effective compact electron beam sources remains a long-standing objective of the efforts to develop the accelerator systems needed for on-site research and industrial applications ranging from electron beam welding to high performance $\mathrm{x}$-ray and gamma ray light sources for element-resolved microanalysis and national security. The need in these applications for simplicity, reliability, and low cost has emphasized solutions compatible with the use of the long established and commercially available pulsed microwave rf sources and L-, S- or X-band linear accelerators. Thermionic microwave electron guns have proven to be one successful approach to the development of the electron sources for these systems providing high macropulse average current beams with picosecond pulse lengths and good emittance out to macropulse lengths of 4-5 microseconds. But longer macropulse lengths are now needed for use in inverse-Compton x-ray sources and other emerging applications. We describe in this paper our approach to extending the usable macropulse current and pulse length of these guns through the use of thermal diffusion to compensate for the increase in cathode surface temperature due to back-bombardment.
\end{abstract}

DOI: 10.1103/PhysRevSTAB.17.120402

PACS numbers: 29.25.Bx, 44.10.+i, 05.45.-a

\section{BACKGROUND}

Microwave thermionic electron guns were developed at Stanford in the early 1980's in response to the need for a source of high peak current electron bunches operable at gigahertz rep rates with emittances adequate for operation throughout the infrared and visible spectral regions. The design developed by Glen Westenskow and John Madey (Fig. 1) proved highly successful in this application, serving as the basis of a compact free-electron laser (FEL) system [1] well suited for both fundamental FEL research at wavelengths extending to $350 \mathrm{~nm}$ in the visible [2] as well as applications in medicine and materials science including the first use of an FEL in human surgery [3]. The design of the Stanford prototype gun was also incorporated as part of the first successful commercially produced FEL light source.

The present capabilities of the thermionic microwave electron gun used at the University of Hawaii (UH) to provide the electrons for subsequent acceleration to energies from 30 to $45 \mathrm{MeV}$ and use in the lab's free electron laser sources are summarized in Table I. As the emphasis in the development of this gun was on high macropulse peak and average currents, the emittance cited in Table I could

\footnotetext{
jeremymk@hawaii.edu

Published by the American Physical Society under the terms of the Creative Commons Attribution 3.0 License. Further distribution of this work must maintain attribution to the author $(s)$ and the published article's title, journal citation, and DOI.
}

undoubtedly be improved by further development of the design if needed for future research. But the gun's measured geometric emittance at $42.2 \mathrm{MeV}$ of $0.105 \mu \mathrm{m}$ and $0.120 \mu \mathrm{m}$ (a normalized emittance of $8.67 \mu \mathrm{m}$ and $9.91 \mu \mathrm{m})$ in the vertical and horizontal planes, respectively [4], has proven adequate for all efforts to date including the pioneering measurements of the sub-Poisson fluctuations of the MkIII FEL's coherent spontaneous harmonic radiation at a wavelength of $350 \mathrm{~nm}$, deep in the blue [2].

The principle limitations of this injector follow from the transient heating of the surface of its lanthanum hexaboride cathode due to bombardment by the electrons emitted late in each rf cycle that reverse their direction in the gun cavity and deposit the energy they gather from the reversed accelerating field in the surface of the cathode. As related in further detail below, the increased cathode current due to this back-bombardment effect reduces the amplitude of the accelerating field as a function of time during each macropulse, changing both the phase and bunch length of the micropulses injected into the subsequent linac. As reviewed at further length below, the tolerable limits on these changes in bunch length and phase determine the allowable increase in cathode current during each macropulse.

Although these effects of increased beam loading due to back-bombardment can in principle be limited by lowering the $Q$ of the gun cavity to reduce the change in shunt impedance due to the change in cathode current, it would be preferable from the standpoint of system efficiency to limit the change in cathode surface temperature by reducing 




FIG. 1. Schematic (top) and photo (bottom) of the upgraded Stanford prototype microwave thermionic gun assembly installed as part of the high average current $45 \mathrm{MeV}$ linac at UH.

or compensating for the effects of back-bombardment. We have developed the technique of "laser cooling" to achieve this objective as described in Sec. IV.

But it seems best to introduce this discussion with an account of the effects of the transient beam loading of the accelerating gradient in thermionic microwave guns on the length and phase of the bunches they provide for injection into the subsequent linear accelerator.

TABLE I. Table of accelerator parameters and measured electron beam properties following acceleration to $42 \mathrm{MeV}$.

\begin{tabular}{lc}
\hline \hline Operation frequency: & $2.856 \mathrm{GHz}$ \\
Energy range: & $30-45 \mathrm{MeV}$ \\
Fractional energy spread: & $0.25 \% \mathrm{FWHM}$ \\
Macropulse length: & $5 \mu \mathrm{s}$ \\
Macropulse average & $200 \mathrm{~mA}$ \\
$\quad$ current: & \\
Bunch length: & $3.1 \mathrm{ps}$ FWHM \\
Geometric emittance: & vertical: $0.105 \mu \mathrm{m}$, horizontal: $0.120 \mu \mathrm{m}$ \\
Normalized emittance: & vertical: $8.67 \mu \mathrm{m}$, horizontal: $9.91 \mu \mathrm{m}$ \\
\hline \hline
\end{tabular}

\section{BUNCHING IN THE THERMIONIC ELECTRON GUN}

Integration of the electrons' equations of motion in the time varying accelerating field of a microwave gun cavity yields the functional relation between the momentum and time at which the electrons exit the gun cavity as shown in Fig. 2 [5]. In the family of guns developed following the demonstration of the Stanford prototype, the electrons which are emitted early in the rf cycle leave the cavity with the highest momentum, decreasing monotonically for later times of emission. Most significantly, the relationship between exit time and momentum is nonlinear, and not easily represented by a low order power series expansion.

Compression of the long "chirped" electron pulses available at the exit of the gun cavity to the picosecond durations required for FEL operation require the inclusion in the transport system from the gun cavity to the subsequent linac the means to introduce a transit time that increases with increasing momentum, and also the means needed to adjust the nonlinear dependence of transit time on momentum to cancel the nonlinear dependence evident in the plot in Fig. 2.

In the family of gun systems that followed the development of the Stanford prototype, these objectives were accomplished by the inclusion of an Enge alpha magnet [6] with an adjustable magnetic field gradient, and a pair of simple drift tubes of adjustable length.

In a simple drift tube of length $l$, the transit time varies with momentum $(\gamma \beta m c)$ as



FIG. 2. Computed times of exit and final momenta of the electrons extracted from the thermionic cathode in the optimized single cell Stanford prototype microwave gun cavity as a function of the phase of emission from the cathode for a peak accelerating gradient of $550 \mathrm{kV} / \mathrm{cm}$. 


$$
\text { transit time }=\left(\frac{l}{c}\right)\left[\left(\frac{1}{\beta \gamma}\right)^{2}+1\right]^{1 / 2}
$$

while in an Enge alpha magnet the transit time for an electron injected at the mirror angle of 40.70 degrees varies as [7]

$$
\text { transit time }=\frac{0.1905\left(\mathrm{~T}^{\frac{1}{2}} \mathrm{~m}^{\frac{1}{2}}\right)}{c \sqrt{\nabla \mathbf{B}}}\left[2+(\beta \gamma)^{2}+\left(\frac{1}{\beta \gamma}\right)^{2}\right]^{1 / 4}
$$

yielding a net transit time through this combination of components given by

$$
\begin{aligned}
\text { net transit time }= & \left(\frac{l}{c}\right)\left[\left(\frac{1}{\beta \gamma}\right)^{2}+1\right]^{1 / 2}+\frac{0.1905\left(\mathrm{~T}^{\frac{1}{2}} \mathrm{~m}^{\frac{1}{2}}\right)}{c \sqrt{\nabla \mathbf{B}}} \\
& \times\left[2+(\beta \gamma)^{2}+\left(\frac{1}{\beta \gamma}\right)^{2}\right]^{1 / 4}
\end{aligned}
$$

The Enge alpha magnet in this system provides the critical means needed to compensate for the time delay in the appearance of the lower momentum electrons at the output of the gun cavity. For normalized momenta in the vicinity of $\beta \gamma \sim 2$, the differing nonlinear dependencies of transit time on momentum in the alpha magnet and the drift tube provide the means needed to independently adjust the low order

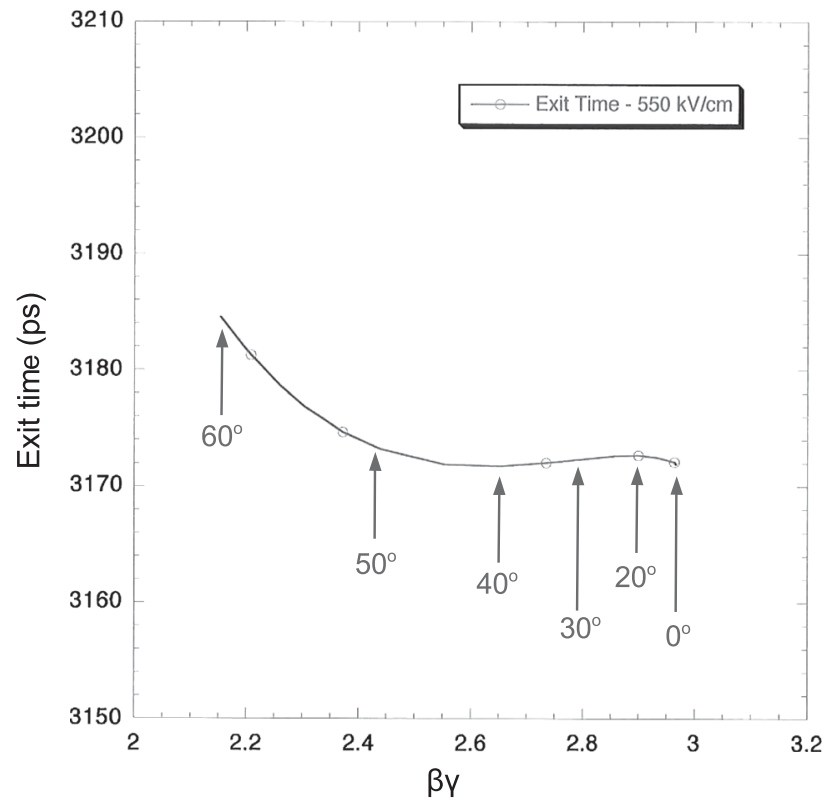

FIG. 3. Computed distribution in momentum and time of the electrons from the prototype Stanford single cell gun cavity as per Fig. 2 as injected into the subsequent linac section after passage through the drift tubes and Enge alpha magnet in the optimized prototype Stanford gun system. The gradient of the magnetic field in the alpha magnet in this simulation was set to $1.29 \mathrm{~T} / \mathrm{m}$. Note that all the electrons emitted from the cathode at phases between 0 and 50 degrees have been compressed into a bunch less than 1 picosecond wide. nonlinear terms relating transit time and momentum for the composite system to almost perfectly compensate for the nonlinear dependence of exit time on momentum out to emission phases of 50 degrees as shown in Fig. 3.

Such fortuitous cancellation of the nonlinear terms inherent in the functional relationship between exit time and momentum is possible only in the region of electron momentum in which the electron velocity remains dependent on momentum. While this is a natural region for operation of single cell microwave gun systems, corresponding to exit electron energies of the order of $1 \mathrm{MeV}$, microwave gun systems with a greater number of cells and higher exit energies can prove less effective in their capability to deliver the very short picosecond pulses available from the family of guns based on the design of the single cell Stanford prototype.

But the nearly ideal compression of the chirped bunches emerging from the gun system as illustrated in Fig. 3 is possible only for a harmonically varying field with the amplitude of $550 \mathrm{kV} / \mathrm{cm}$ as cited in Fig. 2.

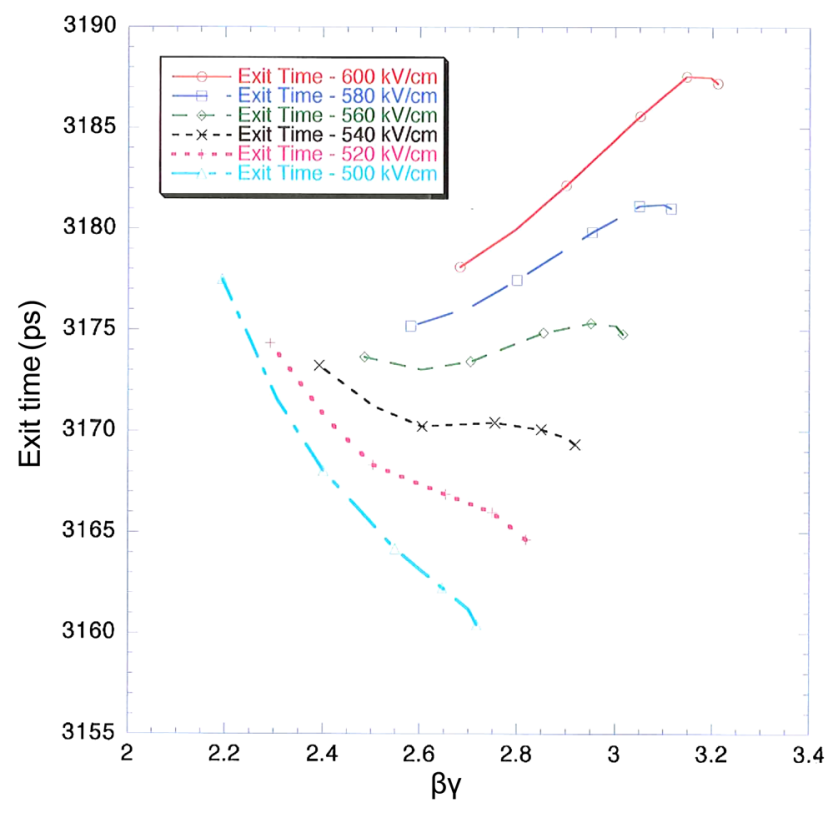

FIG. 4. Computed distributions in momentum and time for the optimized prototype Stanford thermionic gun system for peak accelerating gradients between 500 and $600 \mathrm{kV} / \mathrm{cm}$ and a alpha magnet field gradient of $1.29 \mathrm{~T} / \mathrm{m}$. Note that bunching comparable to that shown in Fig. 3 at a gradient of $550 \mathrm{kV} / \mathrm{cm}$ can be obtained for all gradients between 540 and $560 \mathrm{kV} / \mathrm{cm}$. Electrons with momenta outside the region of "good bunching" were blocked from passage through the alpha magnet by a movable knife-edged beamstop located at the apogee of their orbits through the alpha magnet. The noncritical dependence of bunching on accelerating gradient evidenced in this figure has made it possible to preserve the width of the bunches injected into the linac even as the time-dependent beam loading due to back-bombardment lowered the gradient from an initial value in the vicinity of $560 \mathrm{kV} / \mathrm{cm}$ to a value in the vicinity of $540 \mathrm{kV} / \mathrm{cm}$ at the end of each rf pulse. 
Changes in the accelerating gradient in the gun cavity change both the degree of bunching that can be achieved with a given alpha magnet gradient and also the time at which the compressed bunches are delivered to the first cells of the subsequent linac as shown in Fig. 4. As is apparent from this figure, the accelerating gradient must be maintained between 540 and $560 \mathrm{kV} / \mathrm{cm}$ to keep the duration of the bunches below 3 ps assuming the low energy knife edge in the alpha magnet is set to reject electrons with $\beta \gamma<2.5$.

The rejection of electrons emitted after 50 degrees (with $\beta \gamma<2.5$ ) in the Stanford family of guns was accomplished through the installation of a movable knife edge in the alpha magnet that intercepted the trajectories of the lower momenta electrons, blocking their passage through the system as shown in Fig. 1.

\section{LIMITATIONS IMPOSED BY BACK-BOMBARDMENT}

The increase in beam loading due to the increase in cathode current due to back-bombardment during each macropulse reduces the accelerating gradient during each pulse to values that must be limited to the range in which useful bunching can be achieved as per Fig. 4 . The change in bunch lengths apparent in Fig. 4 between accelerating gradients of 540 and $560 \mathrm{kV} / \mathrm{cm}$ is representative of the largest acceptable change in bunch length for the FEL systems with which these microwave thermionic guns have been used, leading to measured FWHM durations of the accelerated bunches at $\sim 3$ ps as shown in Fig. 5 [8].

The change in cathode current that can be tolerated given these constraints on the change in accelerating gradient can be measured by measuring the change in the electrons momentum distribution during each pulse using the alpha magnet and its low energy knife edge as a spectrometer, and relating these measured spectra to the momentum distributions plotted in Fig. 4 for each accelerating gradient. For the updated version of the Stanford prototype gun now operated at $\mathrm{UH}$, careful measurements of these time dependent momentum distributions have established that the change in cathode current during each macropulse must be limited to no more than $150 \mathrm{~mA}$ to maintain the accelerating gradient between 540 and $560 \mathrm{keV}$ during each macropulse.

The time at which the compressed bunches were injected into the linac also changes with beam loading, with the electrons accelerated by a $540 \mathrm{kV} / \mathrm{cm}$ field reaching the linac approximately 4 ps later than the electrons accelerated by a $560 \mathrm{kV} / \mathrm{cm}$ field. Though not ideal, this effective phase slew appears to the FEL systems driven by the gun and linac as a small change in the $2856 \mathrm{MHz}$ rep rate of the accelerated electrons which can be compensated by adjusting the spacing of the cavity mirrors. Although this shift in phase also shifts the positions of the injected bunches on the peak of the accelerating wave in the linac used to raise



FIG. 5. Temporal electron distribution within a single bunch after acceleration through the linac obtained using the Hamamatsu 5680 streak camera with synchroscan unit. This plot is the average of 100 images of the same single bunch within the macropulse of the $2.856 \mathrm{GHz}$ train of pulses generated by the present UH microwave gun. After subtraction in quadrature of the 2 ps of width attributable to the finite resolution of the streak camera used in these measurements, the 3.7 ps FWHM measured by the streak camera yields an actual bunch FWHM of 3.1 ps.

their energy to $40 \mathrm{MeV}$, the effect on the measured energy spectrum was small. As shown in Fig. 6, the energy spread of the accelerated electrons at the output of the linac at UH is $150 \mathrm{keV}$, closely matching the electrons' energy spread out of the gun and alpha magnet.

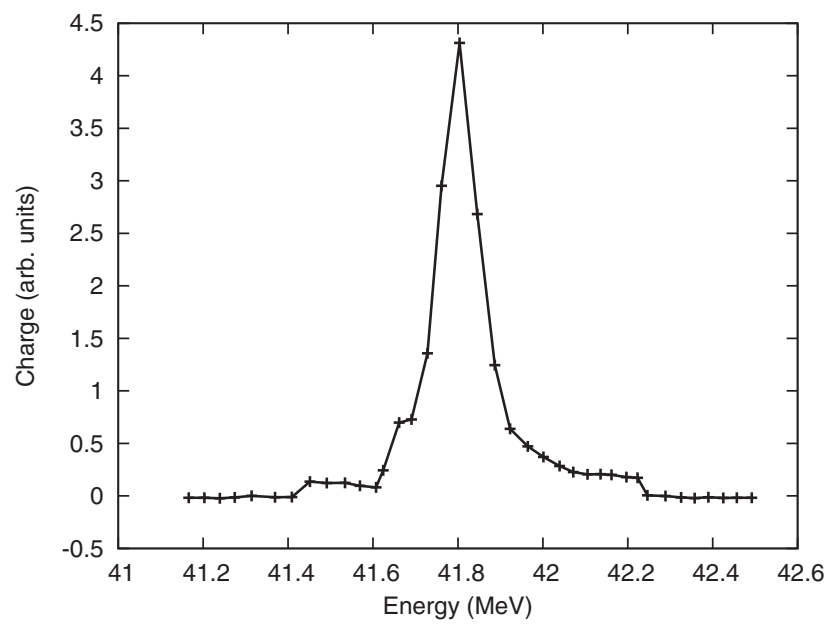

FIG. 6. Measured energy spectrum of the accelerated bunches from the present UH thermionic microwave gun when operated with an accelerating gradient ranging from 600 to $500 \mathrm{kV} / \mathrm{cm}$ as determined by back-bombardment with a gun cavity output current of $500 \mathrm{~mA}$ and an accelerated beam current of $180 \mathrm{~mA}$ at a final energy of $41.8 \mathrm{MeV}$. In this figure, the width of the energy scan is $2.0 \%$, and the FWHM of the energy spectrum is $0.25 \%$, closely matching the electrons' $150 \mathrm{keV}$ energy spread when injected into the linac. 
The final problem presented by the time-dependent temperature rise due to back-bombardment is the increase in current that would be injected into the subsequent linac if all of the electrons emitted by the cathode were to be injected into the linac following bunching. That increase in current, which typically approaches $20 \%$ during operation of the present UH gun cavity, would result in an independent energy slew in the linac as the linac's accelerating field gradient drooped in response to the beam loading attributable to the ramp in accelerated current. The magnitude of this droop in energy would approach $1 \mathrm{MeV}$ under typical operating conditions of $40 \mathrm{MeV}$, far more than could be tolerated for the applications in which this system is used.

In practice, this effect can be nulled by setting the knifeedged beamstop in the alpha magnet that blocks the passage through the alpha magnet of the low energy electrons that exit from the gun cavity to a position that allows a decreasing fraction of the higher energy electrons to pass through the alpha magnet as their energy droops due to increased beam loading in the gun cavity. The relatively weak dependence of bunching on magnetic field gradient significantly facilitates the setups needed to achieve this result, allowing the gradient and resultant orbit sizes in the alpha magnet to be empirically adjusted to yield a nearly flattop current pulse as measured at the output of the alpha magnet.

By these means, it has been possible to exploit the extraordinary nonlinear bunch compression mechanisms inherent in the design of single cell microwave thermionic guns to achieve the high peak and average currents required for effective FEL operation at infrared $[9,10]$ and visible wavelengths [11] including the abilities to explore both the properties of advanced FEL systems including guiding as well as a number of key applications in photochemistry and surgery [3]. This approach to ebeam generation and acceleration also now appears to constitute one of the best available choices for use in high average power inverse Compton X-ray and gamma ray generation systems [12].

But it would remain a major step forward to devise and demonstrate a means to suppress the effects of cathode surface heating due to the back-bombardment effect. In the following section we describe the means available to accomplish this objective, and the experimental data acquired to date in the effort to validate this approach.

\section{USE OF A LASER PREPULSE TO STABILIZE THE CATHODE SURFACE TEMPERATURE}

Previous measurements of the cathode surface temperature of our microwave thermionic gun at $\mathrm{UH}$ show a rise of nearly $60 \mathrm{~K}$ during a typical $5 \mu$ s long macropulse [13], introducing the three problems discussed in Sec. III. The question to be answered is: Does a method exist that can either prevent or compensate for this temperature rise in a microsecond time frame? The $1 \mathrm{~mm}$ long, $3 \mathrm{~mm}$ diameter $\mathrm{LaB}_{6}$ cylindrical cathode in the $\mathrm{UH}$ gun is maintained just above $1700 \mathrm{~K}$ by a thin tungsten filament just behind it, but due to the thermal mass of the cathode and support structure, minimal though it is, the temperature cannot be changed on microsecond time scales with that filament. A more sophisticated contact heating/cooling system is impractical due to the need to keep the assembly surrounding the cathode (and therefore penetrating in to the gun cavity) to an absolute minimum to avoid perturbing the accelerating field. However, a noncontacting method that uses diffusion of heat from the surface of the cathode as a cooling mechanism is feasible.

If the cathode is maintained at a "standby" temperature below the $\sim 1700 \mathrm{~K}$ operating temperature, a laser prepulse with duration in the microsecond regime can be used to transiently increase the cathode surface to a value above the operating temperature, leaving a large temperature gradient between the surface and bulk of the cathode. This temperature gradient leads to rapid cooling of the surface as the heat diffuses into the bulk.

If this laser prepulse deposits the appropriate amount of energy on the cathode surface and is timed just before the rf pulse-and the subsequent heating induced by backbombardment $(\mathrm{BB})$ as the gun emits current- then the diffusive cooling can counteract the heating due to BB and the cathode surface can remain at a stable temperature. This is the laser cooling concept described by Madey [14]. With the temperature stable, the current would no longer increase with macropulse time, the beam loading would be constant, and the accelerating field in the gun cavity would also be constant: eliminating the phase and energy slew and allowing for much longer macropulse times.

\section{A. Numerical model of surface temperature stabilization}

The temperature of the cathode in such a system is a complex function of the laser energy and timing, radiative cooling, and back-bombardment and is further complicated by positive feedback: an increased cathode temperature leads to more thermionic current emission, which leads to more back-bombardment, which increases the cathode temperature. Numerical calculations were performed of the cathode surface temperature taking into account all relevant phenomenon to determine the parameters of the laser prepulse. The basic formulation of this one-dimensional numerical model is described in a reference [15], with the back-bombardment power as a function of depth into the cathode calculated using PARMELA [16], though we have made several improvements: the model of the current emitted by the gun now accounts for space charge limited emission at low fields (Child's law) [17], transitioning to the field assisted thermionic emission, or Schottky equation, when the current becomes thermionically limited [18], and the accelerating field is now appropriately reduced due to beam loading at higher currents using a shunt resistance in the circuit model of 

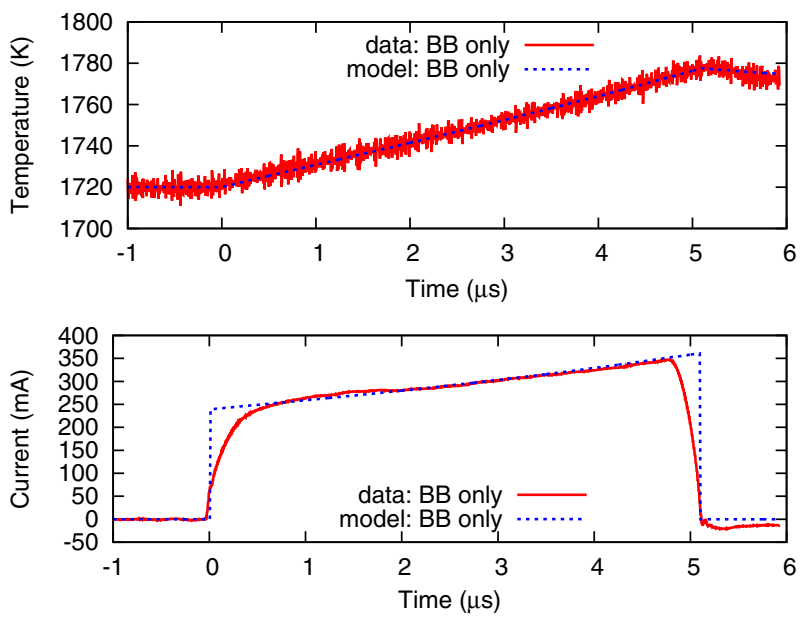

FIG. 7. Numerical model fit of cathode temperature and gun current data for a typical pulse without laser cooling. The red solid curve shows measured data while the blue dashed curve shows results of the numerical model.

the cavity [7]. This improved model was fit to simultaneous gun current and cathode temperature data via the work function of the $\mathrm{LaB}_{6}$ cathode (with measured values in the literature between 2.4 [19] and $2.66 \mathrm{eV} \mathrm{[20]),} \mathrm{resulting} \mathrm{in} \mathrm{a}$ final fit value of $2.485 \mathrm{eV}$, and via the shunt resistance in the circuit model of the cavity which was used to match the change in current due to beam loading. A value of $29 \mathrm{~A} / \mathrm{cm}^{2}$ was used for the Richardson constant [20]. Results of the model fit to data are shown for short time scales in Fig. 7.

Since it appears that the simulated temperature curve does not drop as rapidly as the measured temperature after BB stops at $6 \mu \mathrm{s}$ in Fig. 7, we show comparison of the numerical model and measurement for longer times after
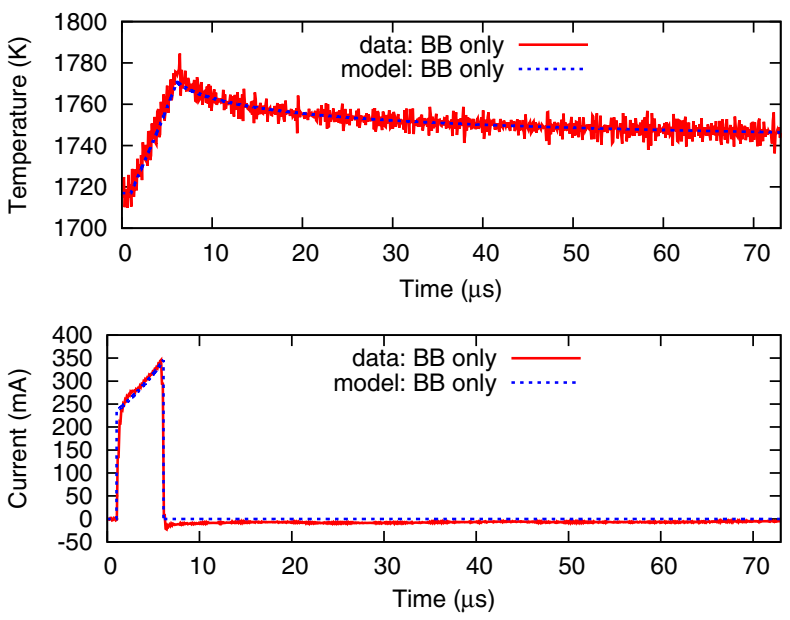

FIG. 8. Numerical model comparison to cathode temperature and gun current data for a typical pulse without laser cooling, showing times long after the accelerating field has been turned off (and BB has ceased). The red solid curve shows measured data while the blue dashed curve shows results of the numerical model.
BB stops in Fig. 8 which confirm that the model is a good fit to measurement for all times except near the rf turn-off. The numerical model does not account for the fill/empty time of the rf cavity during turn-on/-off which is most likely the cause for this discrepancy.

In contrast to the steep rise in current shown in Figs. 7 and 8 , limiting the pulse length to $5 \mu \mathrm{s}$, the numerical model shows that by using a laser prepulse which deposits $329 \mathrm{~mJ}$ (average fluence of $F=4.65 \mathrm{~J} / \mathrm{cm}^{2}$ ) of heat onto the cathode surface over $7 \mu$ s duration just before the $\mathrm{rf}$ pulse enables pulses up to $29 \mu$ s, illustrated in Fig. 9, while keeping the current in same range as we now operate and maintaining the surface temperature of the cathode below $2287 \mathrm{~K}$-well below the melting point of $\mathrm{LaB}_{6}$ of $2483 \mathrm{~K}$ [20]. This is nearly a factor of 6 longer than the $5 \mu$ s pulses we currently operate with.

\section{B. A proof of principle experiment}

The most common pulsed lasers capable of $\sim 500 \mathrm{~mJ}$ pulse energies have durations in the tens of nanoseconds, so they are not ideally suited for this purpose. The short pulses lead to a much higher surface temperature and the formation of a laser induced plasma just outside the surface. Nonetheless, we attempted to test the laser cooling concept with a $q$-switched Nd:YAG laser we had in hand both at the fundamental $1064 \mathrm{~nm}$ wavelength with $15 \mathrm{~ns}$ FWHM pulses, and at the second harmonic $532 \mathrm{~nm}$ with $20 \mathrm{~ns}$ pulses. To verify that these laser wavelengths
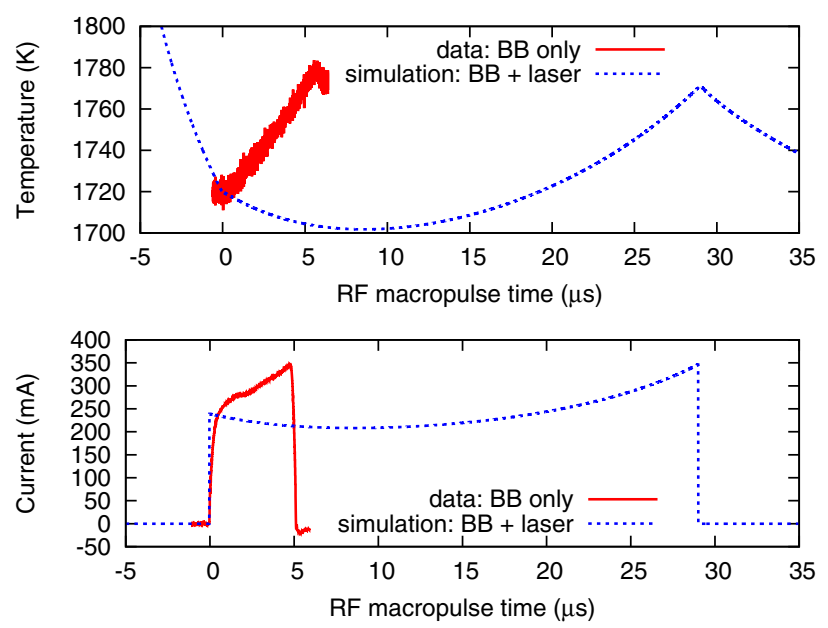

FIG. 9. Numerical model of laser cooling showing cathode surface temperature and gun current as a function of time. The red solid curve, measured data of a typical pulse without laser cooling, shows a steep temperature increase from back-bombardment $(\mathrm{BB})$ when the rf accelerating field is applied at $0 \mu \mathrm{s}$. The blue dashed curve, results of the numerical model with laser cooling, shows the stabilized temperature from the combination of $\mathrm{BB}$ and the cooling effect of $329 \mathrm{~mJ}$ (average fluence of $F=4.65 \mathrm{~J} / \mathrm{cm}^{2}$ ) of heat absorbed on the cathode surface over a $7 \mu$ s long laser pulse initiated $15.5 \mu$ s before the rf field is applied at $0 \mu \mathrm{s}$. 
would be absorbed by the $\mathrm{LaB}_{6}$ cathode, we measured the emissivity to be 0.713 at $1064 \mathrm{~nm}$ and 0.687 at $550 \mathrm{~nm}$ [21] (note measurement below $532 \mathrm{~nm}$ was not possible due to equipment limitations). The laser was initially delivered to the cathode via a $1 \mathrm{~mm}$ diameter fiber optic cable, but the large numerical aperture of the fiber lead to the inability to focus the laser beam tightly on the cathode, so very little of the laser pulse energy was coupled as heat to the cathode surface. Also laser damage to the fiber surface at high energy decreased reliability. An evacuated free-space laser transport system was later constructed and allowed for much tighter focusing on the cathode. The final two mirrors were remotely adjustable to allow optimal steering of the laser onto the cathode during operation. Pulses of 1-40 mJ (average fluence of $F=0.01$ to $0.57 \mathrm{~J} / \mathrm{cm}^{2}$ ) at $532 \mathrm{~nm}$ with duration of $20 \mathrm{~ns}$ were directed onto the cathode, and a quadratic minimum and flattening of the ramp in current due to back-bombardment was observed, commensurate with the numerical model results. Figure 10 shows the cathode temperature and gun current with a $4.66 \mathrm{~mJ}$ (average fluence of $F=0.07 \mathrm{~J} / \mathrm{cm}^{2}$ ) pulse incident on the window to the gun cathode during the rf macropulse. At $532 \mathrm{~nm}$, approximately $92 \%$ of that energy is transmitted through
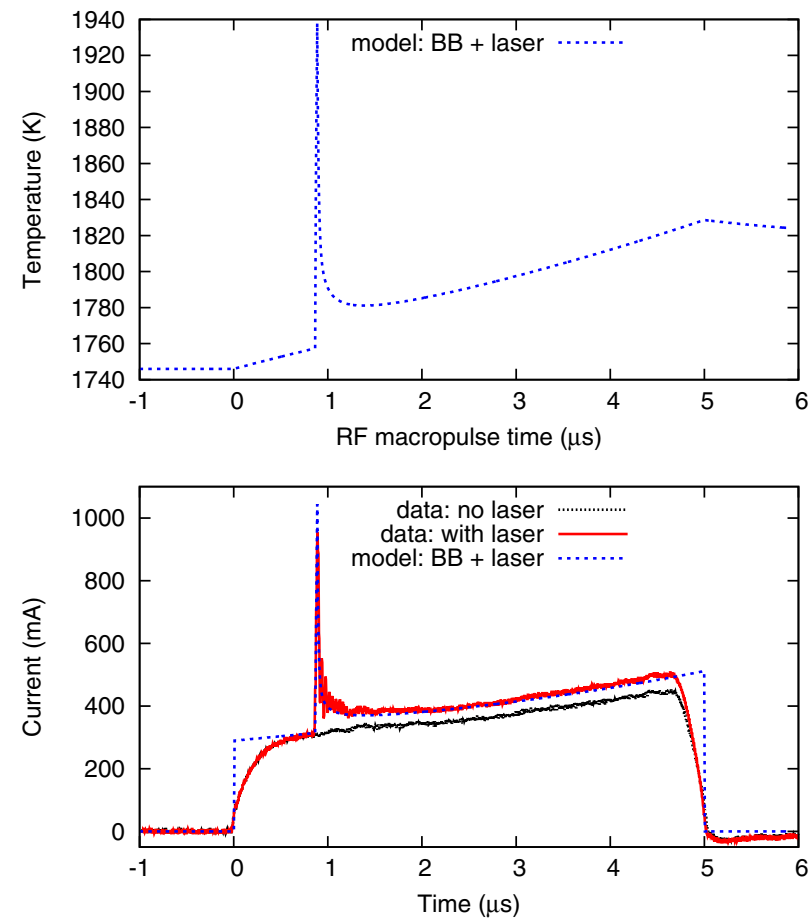

FIG. 10. Electron gun temperature and gun current as a function of time with (red solid curve) and without (black dotted curve) a $4.66 \mathrm{~mJ}$ laser pulse incident on the window to the gun cathode with corresponding numerical model (blue dashed curve). The rf macropulse is active in the gun cavity from 0 to $5 \mu \mathrm{s}$. Temperature data could not be taken simultaneously with the current as the only available optical window to the gun cathode was used for the laser. the window and $68.7 \%$ is absorbed as heat due to the absorptivity (presumed equal to the emissivity) of $\mathrm{LaB}_{6}$.

\section{Limits on peak power of the laser prepulse}

At incident average fluences approaching $0.13 \mathrm{~J} / \mathrm{cm}^{2}$ (9 $\mathrm{mJ}$ total energy), current from the cathode dropped to zero immediately after the laser pulse on every shot of the laser as shown in Fig. 11, presumably due to ionization and plasma formation on the cathode surface and subsequent shorting of the rf cavity. This drop in current was only seen sporadically at incident fluences between 0.13 and $0.04 \mathrm{~J} / \mathrm{cm}^{2}$ and never at fluences below. The numerical model shows that at an incident fluence of $0.28 \mathrm{~J} / \mathrm{cm}^{2}$ (20 mJ total energy), $20 \mathrm{~ns}$ laser pulse would increase the cathode surface temperature to $2490 \mathrm{~K}$, beyond the $2483 \mathrm{~K}$ melting point of $\mathrm{LaB}_{6}$ where evaporation, and therefore plasma formation, are dramatically more likely. The numerical model is only one-dimensional and so only supports a top-hat transverse intensity laser pulse, but the experimental laser pulse is nearly Gaussian in the transverse dimensions, making the peak fluence approximately twice the average fluence, so there is likely a small spot on the cathode that approaches the melting point with a $9 \mathrm{~mJ}$ laser pulse.

\section{Options for limiting laser peak power}

The numerical model has shown previously that a laser with the same energy and longer pulses could avoid plasma formation and still provide the cooling needed [15]. Fortunately, a typical Nd:YAG with tens of nanosecond pulses at the fundamental can be modified to produce

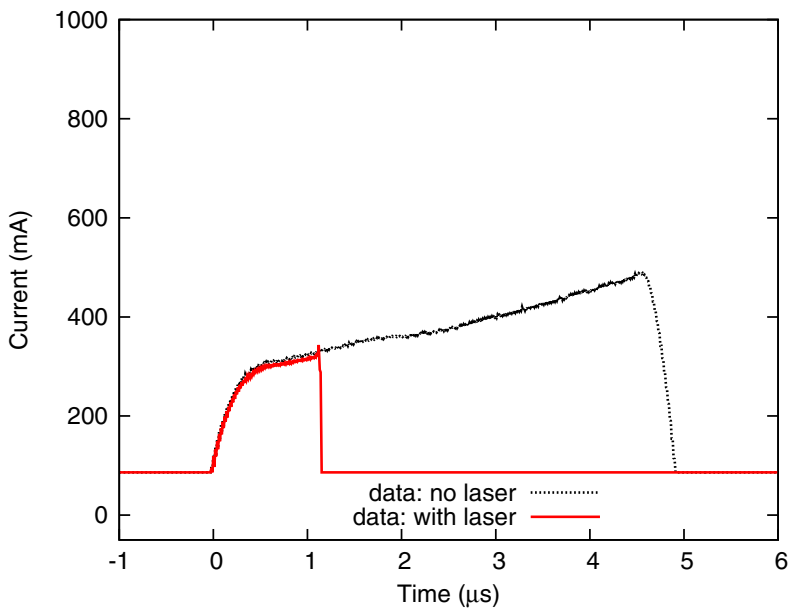

FIG. 11. Electron gun current as a function of time with (red solid curve) and without (black dotted curve) a $20.0 \mathrm{~mJ}$ laser pulse incident on the cathode. The rf macropulse is active in the gun cavity from 0 to $5 \mu \mathrm{s}$. When the laser hits the cathode at $t=1 \mu$ s the gun current immediately drops to zero presumably due to plasma formation and subsequent shorting of the gun cavity. 
pulses of 100 ns to $1 \mu$ s by insertion of a GaAs nonlinear absorber in the oscillator cavity at the Brewster angle [22]. Gallium arsenide has strong two photon absorption at $1064 \mathrm{~nm}$, so it reduces the gain as the laser approaches saturation causing the pulse to build up more slowly and to a lower peak power in the oscillator. A circuit capable of extending the $q$-switch to microsecond duration, which we currently have operational, is also necessary to keep the cavity losses low while the laser builds to saturation. Work is currently in progress at $\mathrm{UH}$ to reproduce the previously demonstrated microsecond laser pulses as required to avoid plasma formation and extend the stabilization of cathode current and temperature to the longer pulse lengths predicted by our model.

Another means to reduce the possibility of ionization and plasma formation is by using the fundamental wavelength of $1064 \mathrm{~nm}$ instead of the $532 \mathrm{~nm}$ used in the experiments to date. The visible wavelength was used initially for convenience before we were aware ionization was an issue, but the longer IR wavelength is less likely to cause ionization [23], so it will be used in future experiments.

The transverse beam profile of the Nd:YAG laser used is pseudo-Gaussian with an average $\mathrm{M}^{2}$ value of 4.11. The beam was focused such that twice the beam waist, $2 w$, was equal to the cathode diameter. A beam homogenizer could be used to achieve more uniform illumination consistent with the assumption of the one-dimensional model. Although the design of the present UH gun cavity does not provide the access needed to include this capability, the cavity could easily be modified to include an aperture for the required dimensions in future versions.

\section{SUMMARY AND CONCLUSIONS}

The microwave thermionic gun concept has largely faded from sight given its perceived limitations on bunch length due to the broad spectrum of energy of emitted electrons and on macropulse length due to back-bombardment. We have shown here that picosecond bunch sizes are attainable with such systems by carefully balancing the effect of electron momentum spread and transit time through the gun system. Further, we have shown a method to counteract back-bombardment heating and increase the available macropulse lengths (and therefore average current) by a factor of 6 .

This predicted performance enhancement is significant for cavity enhanced inverse-Compton $\mathrm{x}$-ray and gamma ray sources that require high average current, high brightness, and high repetition rates for which the thermionic gun is well suited [12]. Cavity enhanced sources offer the most straightforward route to a compact inverse-Compton sources, which is our focus, so we limit the following discussion to those sources.

The new generation of photocathode guns, with energy spreads and emittances far smaller and peak brightnesses far greater than those achievable with the thermionic gun, have enabled the current $\mathrm{x}$-ray light sources like the LCLS and have also been proposed for cavity enhanced inverseCompton (see for example [24]). However, photocathode guns are not the optimal choice for cavity enhanced sources for a number of reasons.

First, since the storage cavity length $L=c /(2 f)$ with pulses stacking on every round-trip, a gigahertz repetition rate permits a cavity with $L=15$ centimeters and centimeter diameter mirrors while the $\sim 100 \mathrm{MHz}$ repetition rates of photocathode guns [24,25] require $L=1.5$ meters and 10 centimeter diameter mirrors: far larger than can be constructed with the necessary surface figure to achieve the required micron-scale focal spots.

Second, $10 \mu$ s pulses from the $2.856 \mathrm{GHz}$ repetition rate, $110 \mathrm{pC} /$ bunch gun at UH delivers over $3000 \mathrm{nC}$ of charge to the optical storage cavity over the $1 / \mathrm{e}$ decay time of the stored optical pulses in the cavity: far greater than the $10 \mathrm{nC}$ that can be delivered typical photocathode guns in the same time $[24,26]$. Recalling the linear average ebeam current dependence of inverse-Compton x-ray average power [27], it is clear that a stabilized thermionic gun is a superior choice in this regard.

Third, in the thermionic gun case, $x$ rays are delivered in a steady stream of modest peak intensity pulses whereas photocathode guns deliver a less frequent high intensity pulse that could lead to pulse pileup in X-ray detectors.

Fourth, while thermionic guns even with laser cooling will still ultimately be limited by the thermal issue of backbombardment, photocathode guns will likely face thermal management problems of their own if operated at gigahertz repetition rates and high macropulse average currents that have yet to be addressed including the possibility of ramps in cathode current due to heating of the cathode (due to its low quantum efficiency (QE) of $1 \%-10 \%[26,28])$ by the incident pump laser beam. That is not to say that these thermal limitations cannot be overcome: indeed the DC photoemissive gun recently developed at Cornell [29] has impressive specifications and is the only photocathode gun we are aware of with similar repetition rates $(1.3 \mathrm{GHz})$, bunch charge $(77 \mathrm{pC})$, and average current $(65 \mathrm{~mA})$. However the complexity and cost of such a system make it impractical for the niche for which we propose the thermionic gun is better suited: cavity enhanced inverse Compton x-ray and gamma ray systems.

Finally, the thermionic gun cathodes are typically more rugged than photocathodes. The $\mathrm{LaB}_{6}$ thermionic gun cathodes have extremely long lifetimes, can be exposed to atmosphere and recover full emission, and can be operated at vacuum levels of $10^{-5}$ Torr [19] where common photocathodes have QEs that decrease with time and cannot tolerate exposure to pressures above $\sim 10^{-10}$ Torr $[26,28]$.

Given these considerations, we believe the thermionic gun is the best choice for cavity enhanced inverse-Compton sources and other applications that require gigahertz 
repetition rates and high average current in a low cost, highly reliable package and its utility in these applications is further enhanced with the improvements elucidated in this note.

\section{ACKNOWLEDGMENTS}

Financial support for this work was provided by the U.S. Department of Homeland Security under Federal Grant Award No. 2010-DN-077-ARI045-05 and No. 2011-DN077-ARI055-04.

[1] G. A. Westenskow and J. M. J. Madey, Laser Part. Beams 2, 223 (1984).

[2] T. Chen and J. Madey, Nucl. Instrum. Methods Phys. Res., Sect. A 407, 203 (1998).

[3] S. V. Benson, J. M. Madey, J. Schultz, M. Marc, W. Wadensweiler, G. A. Westenskow, and M. Velghe, Nucl. Instrum. Methods Phys. Res., Sect. A 250, 39 (1986).

[4] P. Niknejadi (to be published).

[5] We have neglected space charge effects in these calculations since the current is only space charge limited up to $\sim 3$ degrees, or $\sim 3 \mathrm{ps}$, into the emission cycle after which the current becomes thermionically limited (as determined using the model in Sec. IVA) and space charge has little effect.

[6] H. A. Enge, Rev. Sci. Instrum. 34, 385 (1963).

[7] G. A. Westenskow, J. M. J. Madey, L. C. Vintro, and S. V. Benson, Stanford HEPL Technical Note No. TN-86-1, 1986.

[8] A. Lumpkin, M. Hadmack, J. Kowalcyzk, E. B. Szarmes, and J. M. J. Madey, in Proceedings of the 35th International Free-Electron Laser Conference, edited by C. Scholl and V. R. Schaa (JACoW, New York, 2013), pp. 325-328, ISBN 9783954501267.

[9] S. V. Benson, J. Schultz, B. A. Hooper, R. Crane, and J. M. Madey, Nucl. Instrum. Methods Phys. Res., Sect. A 272, 22 (1988).

[10] C. Brau, Nucl. Instrum. Methods Phys. Res., Sect. A 319, 47 (1992).

[11] N. Sereno, M. Borland, and A. Lumpkin, arXiv:physics/ 0008116.

[12] J. M. J. Madey, E. B. Szarmes, M. R. Hadmack, B. T. Jacobson, J. M. D. Kowalczyk, and P. Niknejadi, in
Proceedings of the SPIE, X-Ray Nanoimaging: Instruments and Methods, edited by B. Lai, SPIE Proceedings Vol. 8851 (SPIE-International Society for Optical Engineering, Bellingham, WA, 2013).

[13] J. M. D. Kowalczyk, M. R. Hadmack, and J. M. J. Madey, Rev. Sci. Instrum. 84, 084905 (2013).

[14] J. M. Madey, U.S. Patent No. 2011-0248651, 2011.

[15] J. M. D. Kowalczyk, M. R. Hadmack, and J. Madey, in Proceedings of the 35th International Free-Electron Laser Conference, edited by C. Scholl and V. R. Schaa (JACoW, New York, NY, 2013), pp. 79-81.

[16] C. B. McKee, Ph.D. dissertation, Duke University, 1994.

[17] C. D. Child, Phys. Rev. (Ser. I) 32, 492 (1911).

[18] P. Sprangle, J. Peñano, B. Hafizi, D. Gordon, S. Gold, A. Ting, and C. Mitchell, Phys. Rev. ST Accel. Beams 14, 020702 (2011).

[19] H. Ahmed and A. N. Broers, J. Appl. Phys. 43, 2185 (1972).

[20] J. M. Lafferty, J. Appl. Phys. 22, 299 (1951)

[21] J. M. D. Kowalczyk, M. R. Hadmack, E. B. Szarmes, and J. M. J. Madey, Int. J. Thermophys. 35, 1538 (2014).

[22] A. C. Walker and A. J. Alcock, Rev. Sci. Instrum. 47, 915 (1976).

[23] R. M. Wood, The Power- and Energy-Handling Capability of Optical Materials, Components, and Systems (SPIEInternational Society of Optical Engineering, Bellingham, WA, 2003), p. 36, ISBN 0-8194-4743-9.

[24] Compact X-ray Light Source (Pacific Northwest National Laboratory, Richland, WA, 2012).

[25] B. L. Militsyn, I. Burrows, R. J. Cash, B. D. Fell, L. B. Jones, J. W. Mckenzie, K. J. Middleman, S. N. Kosolobov, H. E. Scheibler, and A. S. Terekhov, in Proceedings of the 11th European Particle Accelerator Conference, Genoa, 2008 (EPS-AG, Genoa, Italy, 2008), pp. 235-237.

[26] F. Sannibale et al., Phys. Rev. ST Accel. Beams 15, 103501 (2012).

[27] G. A. Krafft and G. Priebe, Rev. Accel. Sci. Techol. 03, 147 (2010).

[28] D. Sertore, L. Monaco, and C. Pagani, in Proceedings of the 10th European Particle Accelerator Conference, Edinburgh, Scotland, 2006, edited by C.-L. Biscari, H. C. D. Owen, C. C. Petit-Jean-Genaz, J. C. Poole, and J. C. R. Thomason (EPS-AG, Edinburgh, Scotland, 2006), pp. 2496-2498.

[29] B. Dunham et al., Appl. Phys. Lett. 102, 034105 (2013). 